International Journal of Applied Dental Sciences 2021; 7(1): 148-153

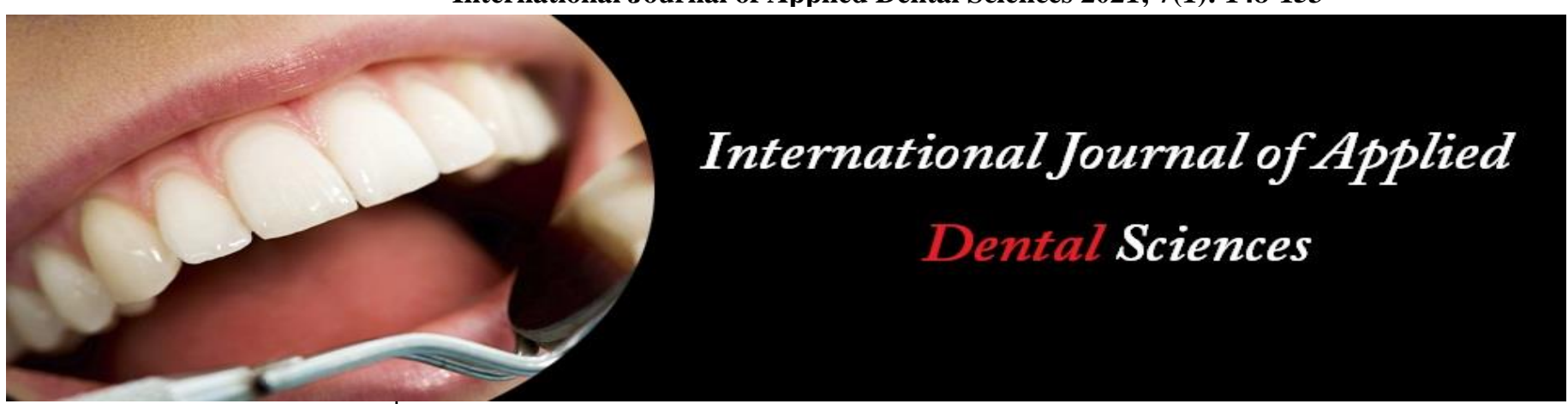

ISSN Print: 2394-7489

ISSN Online: 2394-7497

IJADS 2021; 7(1): 148-153

(C) 2021 IJADS

www.oraljournal.com

Received: 04-11-2020

Accepted: 12-12-2020

Dr. Hind Abdullah Naji B. D. S, \& Dip. IN O.S, Ministry of Health, Department of Health Wasit, Aziziyah General

Hospital, Wasit, Iraq
Corresponding Author: Dr. Hind Abdullah Naji B. D. S, \& Dip. IN O.S, Ministry of Health, Department of Health Wasit, Aziziyah General Hospital, Wasit, Iraq

\section{Intentional replantation: A measure to save the natural tooth}

\section{Dr. Hind Abdullah Naji}

DOI: https://doi.org/10.22271/oral.2021.v7.i1c.1129

Abstract

Context: Intentional replantation (IR): is an accepted procedure in which a tooth is extracted and treated outside the oral cavity, then reinserted into its original socket, this technique can be useful for teeth that cannot be treated with conventional endodontic therapy (or) surgery.

Aim: Preservation of natural dentitions is the primary (aim) of any conservative treatment modality, so IR is one of the techniques used to save the natural tooth.

I treated thirty infected hopeless teeth for thirty patients.

These cases cannot be treated with conventional endodontic therapy (or) surgery and the results were satisfactory.

In these papers I reported four cases had been done according to the same principle (IR) but differ in simple things such as: (the patient complaint, type of crown filling, type of fixation of the tooth, and time of follow up).

First case: A 23-years old male was referred to with a complaint of recurrent pain and discomfort in the mandibular right area. Clinically there was a sinus tract present, associated with the $1^{\text {st }}$ right molar, the crown repair with a large amalgam filling, radiology showed there was per apical radiolucency and root canal filling with unsatisfactory obturation. When the patient selects IR and refused other options, so IR was planned. Clinical and radiograph follow-up 6 months postoperatively revealed no sign or symptoms of infection or inflammation and the tooth retain natural function.

Second case: A 25-years old male, with a complaint of severe pain following the $2^{\text {nd }}$ visit of retreated Root Canal Therapy to the right upper second premolar. The patient refused to continue endodontic treatment and wanted a solution to keep his tooth the other way, after a discussion about the options that help him to keep his tooth, he selects the I R option. So, IR was done followed by clinical examination 7 months postoperatively revealed no signs or symptoms of infection or inflammation.

Third case: A 39-years old female, referred with a persistent dull pain at right upper teeth, clinical and radiological exam shown that there was apical lesion. I R planned after agreement of patient, post-operative follow up revealed no sign or symptom of infection or inflammation.

Fourth case: A 20-years old female, referred a complaint of severe pain in the right mandibular molar, clinically there was a large carious lesion, the gum swollen, radiograph showed that there was an apical lesion around the apical root of $3^{\text {rd }}$ molar. IR was done, after follow up the tooth was healthy and functions.

Keywords: IR (Intentional replantion), R.C.T (root canal therapy), L.C (light cure), S.S (stainless steel), PDL (periodontal ligament)

\section{Introduction}

Intentional replantation (IR) is a concept that has been known for over a thousand years ${ }^{[1]}$ and consists of a technique in which a tooth is intentionally extracted and reinserted into its socket immediately after endodontic treatment and apical repair outside the oral cavity ${ }^{[2]}$

As various investigators report varying success rates, it is difficult to predict the outcome for IR. Bender and Ross man evaluated 31 cases with an overall success rate of $80.6 \%$, (6 cases reported failure). Replanted teeth survived from 1 day to 22 years ${ }^{[3]}$.

Nuzzolese and other ${ }^{[4]}$ state that the success rate of IR at 5 years reported in the literature range from $70 \%$ to $91 \%$.

Chanadra and Bhat reported a case of IR for a mandibular $2^{\text {nd }}$ molar to relieve continuing symptoms, the tooth with no endodontic therapy was carried out either during or after the procedure, they used amalgam for apical seal and then replanted. 
At 20 -years of follow-up, the tooth was healthy and functional with no evidence of root resorption and ankylosis ${ }^{[5]}$.

$\mathrm{Yu}$ and others reported a case where a combined endodontic periodontal lesion on a mandibular $1^{\text {st }}$ molar was treated by IR and application of hydroxyapatite. At a 15-month follow-up examination, the tooth was healthy and functions well ${ }^{[6]}$.

Peer-reviewed 9 cases of IR. only one case of replanting ion showed evidence of pathos is, his report suggests that IR is a reliable and predictable procedure and should be considered more often as a treatment to maintain the natural dentition ${ }^{[7]}$.

\section{History of Teeth Replantation}

The first reported cases of replanted teeth by Pare in 1593

By Pierre in 1706

BY Wigoper in 1933

BY Lenstropandskieller in 1959

BY Anderesen in 1966

BY Cvek in 1974

BY Lindskog in 1977

BY Blomlof in 1980

BY Andreasen in 1981

BY Matsson et al. In 1983

BY Trpeetal in 1992

Indication: Authors mentioned many cases indicated for IR. Such as

1. Cases in which surgical endodontic (re) treatments are not feasible, or have already failed, is an accepted procedure when routine RCT/endodontic surgery is impractical or impossible $e . g$., an obstruction of the canal ${ }^{[8-9]}$.

2. IR can be also considered a suitable treatment for teeth with root perforations that have difficult endodontic or surgical access ${ }^{[10]}$.

3. Generally, IR is not recommended for teeth with periodontal disease [11, 12]; however, it has recently revealed good results for treatment of periodontally involved teeth ${ }^{[13]}$.

4. This method has also been used in the management of vertical fractures and certain anatomical malformations e.g., radicular groove ${ }^{[14]}$.

5. Anatomy difficulty such as a thick bony plat limits access (especially in the lower $2^{\text {nd }}$ premolar $/ 3^{\text {rd }}$ molar), or vital structure such as neurovascular bundles are at risk (especially the mental $\mathrm{N}$ in the region of the lower $1^{\mathrm{st}}$, 2nd molar). Also due to other factors such accessibility, lack of patient cooperation to undertake surgical procedures ${ }^{[15]}$.

Contraindication: Authors mentioned two main contraindications include teeth with flared or curved roots and severe periodontal disease in which there is marked tooth mobility ${ }^{[16]}$.

Advantage of IR: Several studies advocated that IR should be reserved as a "last resort" after other (re) treatment options ${ }^{[17-}$ 18]. An alternative line of thought believes That IR is an economical and conventional technique that is of short duration, and easy Manipulation [19]. Others mentioned less invasive than apical surgery and not the last hope procedure. [20].

A complication of IR: Most studies reported two complications which are inflammatory root resorption (mobility) and ankylosis; due to trauma to the periodontal dental ligament (PDL), and this will be reducing the survival rate of the replanted teeth.
Ankylosis can result if the tooth is outside the oral environment for more than 1 hour ${ }^{[21]}$. The critical event in any reimplantation following extraction of a tooth is the preservation of cellular vitality in a periodontal dental ligament [22]. These complications are directly related to the time the tooth is retained extra orally for treatment; the longer the tooth is kept outside the socket, the poorer the prognosis.

So, in this method, two important things must be found ${ }^{[23]}$.

1. When tooth extracted avoiding unnecessary damages to the PDL

2. Rapid reinsertion into the alveolus immediately after endodontic treatment/apical repair outside the oral cavity.

Update study in 2011, reported that: Most of this concern about the resorptive process and ankylosis come from experience with teeth that have been avulsed. IR is a completely different situation from traumatic avulsion. You could reasonably expect far more trauma to the cementum and bone during a traumatic avulsion, but probably more importantly is that the tooth is often left, contaminated, dry, and out of the socket for an extended period. This led to necrosis of the PDL, and ankylosis ensued. With IR, the tooth is very carefully extracted and maintained out of the mouth in a moist environment for a very limited time. These conditions are ideal to allow healing [24]

\section{Cases Report}

First case report: A 23 - years old male patient was referred to with a complaint of recurrent pain and discomfort in the mandibular right area. clinically there was sinus tract present, associated with the $1^{\text {st }}$ right molar, the crown filled with a large amalgam filling, radiology showed there was apical radiolucency, also there was radiolucency in bifurcation area and root canal filling with unsatisfactory obturation see Pic. (1). Diagnosis of chronic per apical lesion was made.

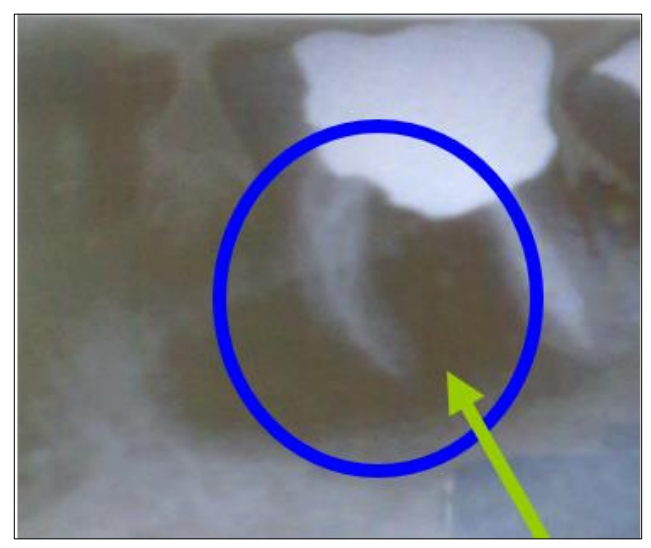

Pic 1: the radiograph showed, there was (periapical, bifurcation) radiolucency and root canal filling with unsatisfactory obturation

\section{The possible treatment options were explained to the patient including}

1. Tooth extraction with (or) without replacement,

2. Endodontic retreatment and post core crown replacement,

3. Peri radicular surgery, and

4. Intentional replantation.

The patient rejected the first three treatment options. Due to previous limitations and the desire of the patient to maintain the tooth, IR was indicated. Before the extraction and reimplantation procedure, the patient had been informed of the possible problems: 
1. Breaking the root itself, which would require extraction and implant or bridge.

2. Breaking of the crown, which would require a new crown.

3. The possibility of infection, inflammation, root resorption, and ankylosis. After an agreement of the patient was gain, IR steps were done.

\section{Clinical Procedure}

1. A chlorhexidine rinse was initiated (3 times daily) one day before the procedure and continued for one-week later control of the oral microflora.

2. Before extraction, the tooth was taken out of occlusion, because when the tooth is repositioned swelling may result in the tooth slightly extruded from the socket, so it's important to ensure good clearance from opposing teeth.

3. After administering local anesthesia, the mandibular first molar was intentionally extracted without any damage to the buccal (or) lingual plates of the alveolar bone see Pic. (2).

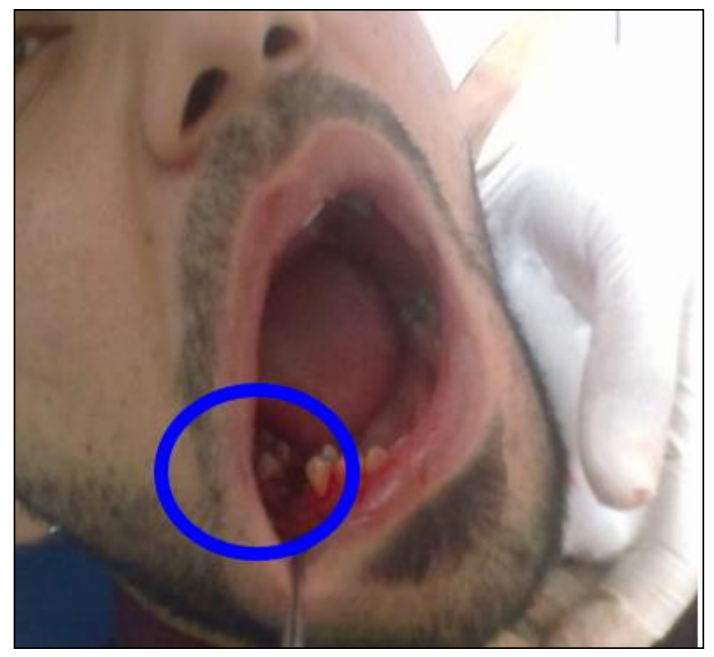

Pic 2: The tooth was extracted without any damage to the buccal (or) lingual plates of the alveolar bone

4. The lesion was removed from bifurcation and apical areas of the root, and the root should be touched as little as possible see Pic. (3)

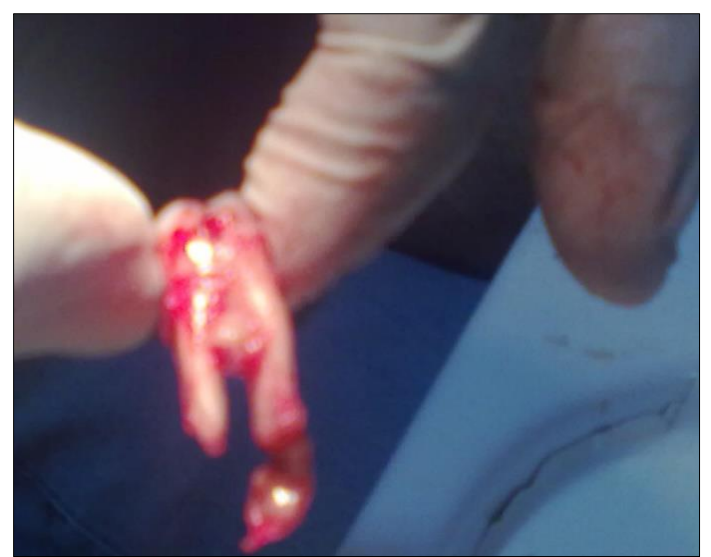

Pic 3: see the size of the lesion which was removed as gentle as possible

5. After root-end resections, $3 \mathrm{~mm}$ deep root-end cavities were prepared by inverted con bar, and the root-ends were filled using an amalgam.

6. Clean the socket to remove the clot and infected tissue, during that the tooth had been kept in wet sterilized gauze, this increases the possibility of PDL vitality. See pic (4)

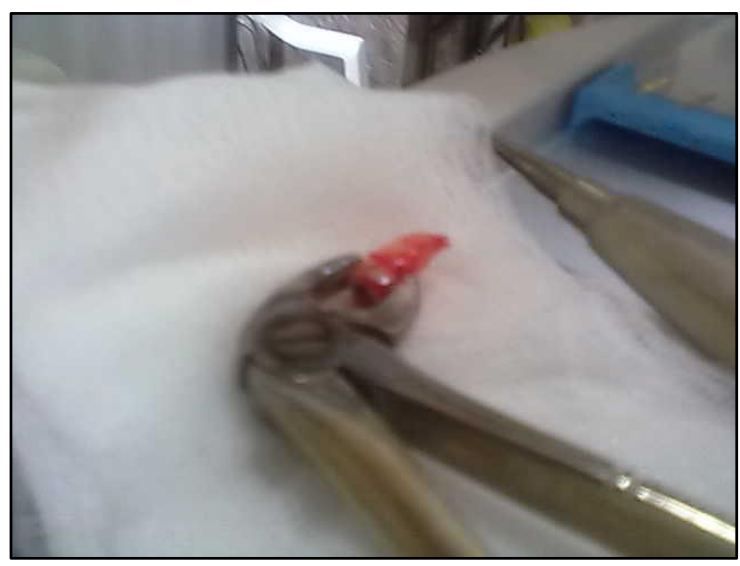

Pic 4: tooth had been kept in a wet sterilized gauze

7. The tooth was then replanted into its socket.

8. Fixed by Stainless steel wire (auther reported fixation should be removed after1weaks to 2 weak); the accurate repositioning was confirmed radiographically. See pig (5)

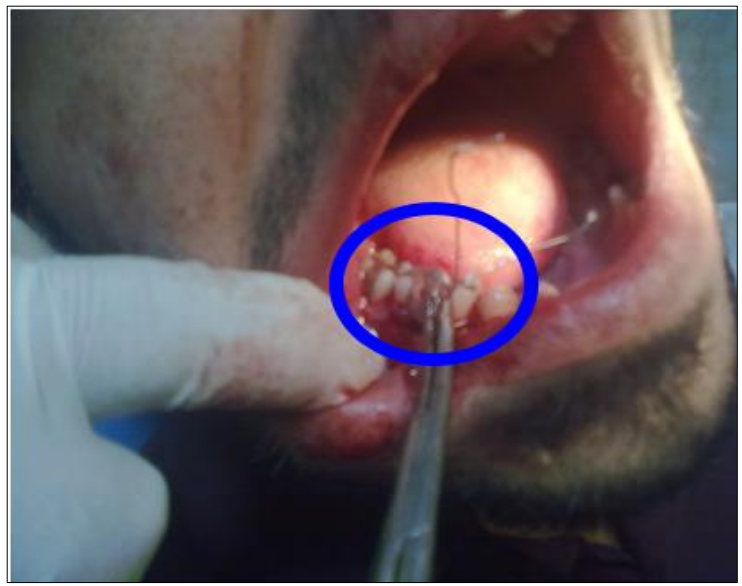

Pig 5: fixation by S.S

The procedure was carried out in a total of 15 minutes. The patient was given postoperative instructions for a soft diet, careful routine oral hygiene, and gave antibiotic with an analgesic. The tooth was inspected 7, 14 days, and 3 weak postoperatively via routine intraoral examinations. At 6 months postoperative sessions there were no clinical signs or symptoms of inflammation (or) infection, no tenderness to percussion or palpitation, no pain or discomfort, sinus tract disappears, the tooth retains to natural. The patient was informed to recall visits to further follow up, also informed to come in any time complication appears. See pic. $(6,7)$

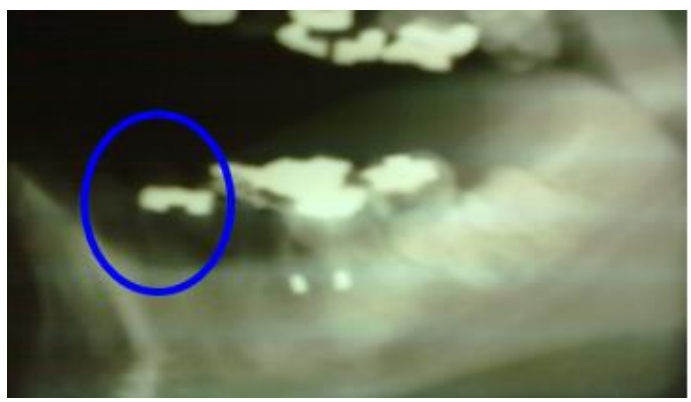

Pic 6: The tooth after 3 months no sign of resorption or ankylosis 


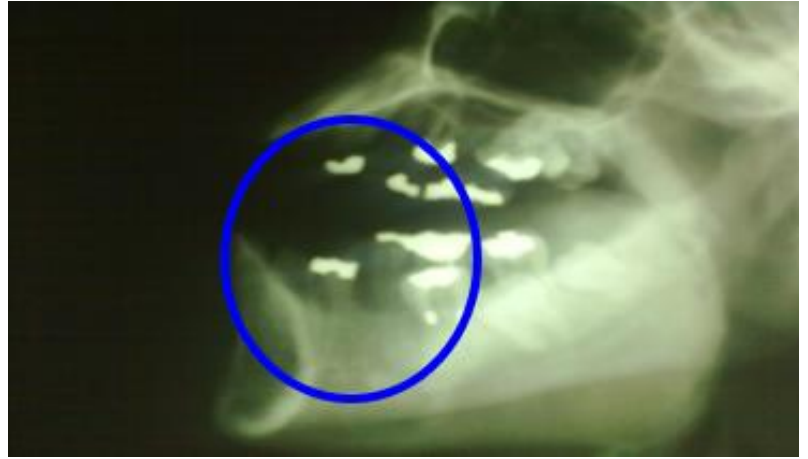

Pic 7: After 2 weak, the tooth with fixation

Second Case: A 25 - years old male, with of complaint of severe pain following the retreated Root Canal Therapy (R.C.T) to the right upper second premolar. Clinically, there was a large disto occlusal (DO) cavity. Radiographic, there was a small periapical lesion. The patient refused to continue endodontic treatment because R.C.T might need multi-visit until the pain subsides and he was could not bear more pain. He was a desire to keep his tooth in another way but if there was no other way to keep it, he would extract it. So other options had been discussed with him, all complications of each option were mentioned. At the end of the discussion, the patient decided to extract the tooth and replantation. Given the patient's desire and difficulty of endodontic surgery because the root near the sinuses so the decision was to employed IR as an alternative method to keep the tooth.

Same previous precaution in $1^{\text {st }}$ case was done, then tooth extracted carefully, the RC-filling did outside the oral cavity, and DO cavity filled with LC filling. After a good apical seal, the tooth reinserted into its cavity and fixed by LC-filling. The procedure was carried out in a total of 30 minutes. An appointment gave to the patient after one weak. Examination after one weak showed no sign or symptom of inflammation, the patient had no pain and very satisfy. Follow up after 7 months, Radiographically, showed there was no root resorption or ankylosis. See pic. (8)

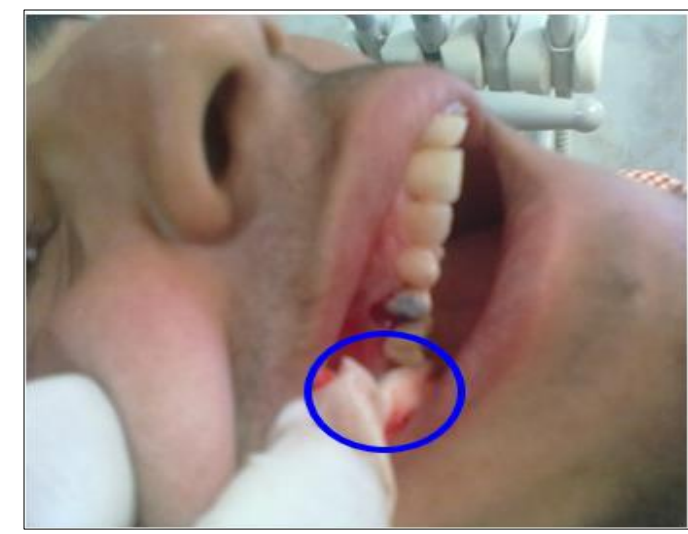

Pic 8: the tooth reinserted to its socket

Third case report: A 39-years old female, referred with a complaint of discomfort and sensitivity in the upper left area. After a radiograph showed that the upper $1^{\text {st }}$ premolar endodontically treated, with over-instrumentation of the R. C. $\mathrm{T}$. A large periapical lesion was observed surrounding the root. Root canal therapy had been completed several years ago. Clinical examination; the tooth was tender to percussion and palpation. The patient had a limitation of mouth opening. Given this limitation and the patient's refusal to undergo a more invasive apical surgery, the intentional replantation technique was indicated as an alternative method to keep her tooth.

Same previous precaution in $1^{\text {st }}$ case was done, then tooth extracted carefully, after good apical seal the tooth reinserted to its cavity and fixed the tooth to the adjacent teeth by glass ionomer cement. The procedure was carried out in a total of 20 minutes an appointment gave to the patient after one weak. Examination after one weak showed no sign or symptom of inflammation, follow up 8 months postoperatively by Radiographic, showed there was no root resorption or ankylosis, clinically there was nothing abnormal notice and the patient had no complaints. see pic $(9,10.11,12)$

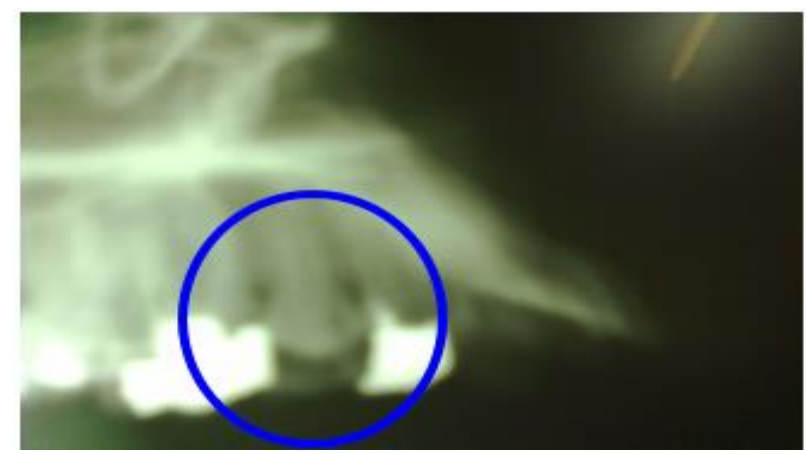

Pic 9: Radiograph shows apical lesion and root canal filling

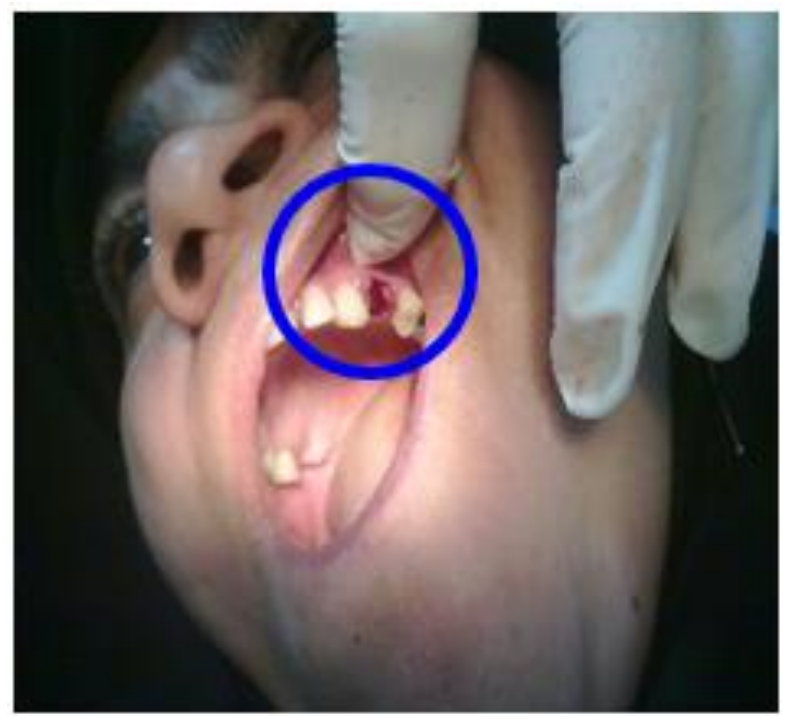

Pic 10: Socket after extract upper first premolar

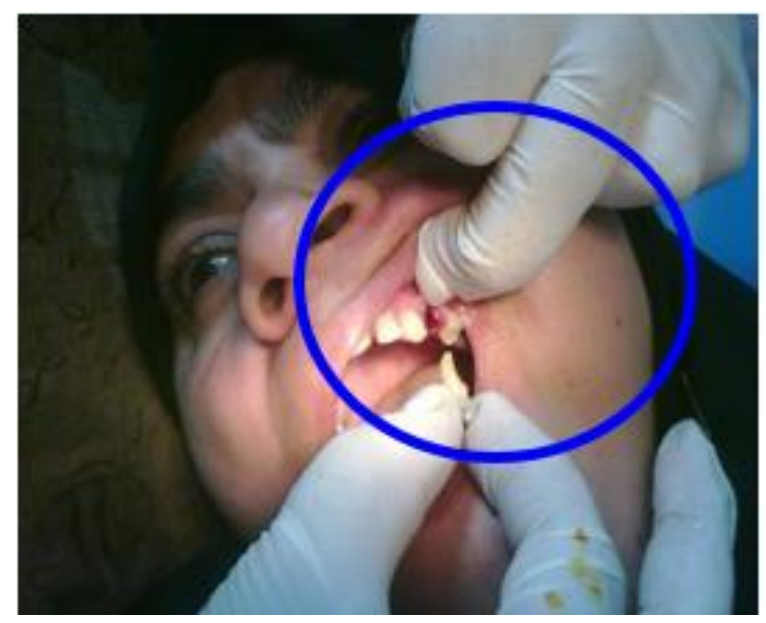

Pic 11: Direction of tooth to replant 


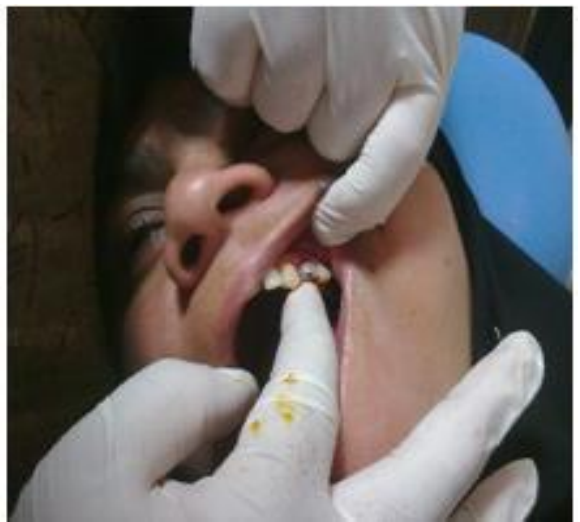

Pic 12: Replant of the tooth in the same socket after treat tooth out side the mouth

The fourth case reports A 25-year-old female referred with a complaint of severe pain in the right mandibular molar, clinically there was a large carious lesion at $3^{\text {rd }}$ molar, the tooth slight tender to percussion, the gum is swollen, the $1^{\text {st }}$ molar was extracted (before long period), the patient had good oral hygiene, radiograph showed that the $3^{\text {rd }}$ molar associated with the apical lesion. The patient desire to keep her tooth, because she did not ready to lose another tooth, as endodontic surgery very difficult in this region, so the decision was RI to retain the tooth to natural function.

Same previous precaution in $1^{\text {st }}$ case was done, then the tooth extracted carefully. In this case, the tooth not filled extra orally with R.C, filling. Just I did a good apical seal to the root with amalgam then the tooth reinserted to its cavity (I follow steps reported by Chandra RV, Bhat KM to manage this case) (see ref, 7) and finally fixed by glass ionomer cement with the adjacent tooth. The procedure was carried out in a total of 25 minutes. Examination after one weak showed no sign or symptom of inflammation. After 2 weeks the soft tissues appeared pink in color with minimal inflammation and pain upon biting had diminished. After 7 months radiographic showed there was no root resorption or ankylosis, the patient had no complaint and the tooth retain natural function.

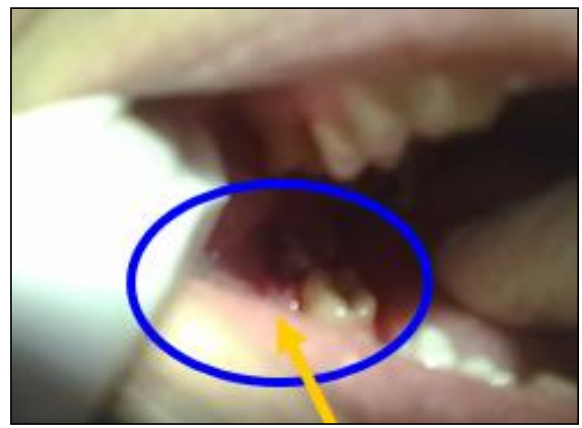

Pic 13: Tooth extraction

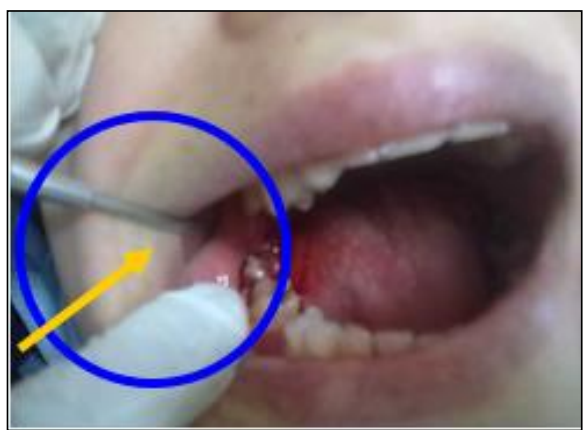

Pic 14: Tooth replantation

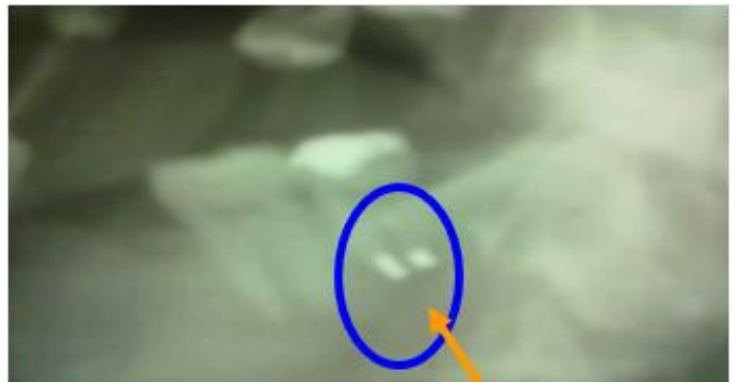

Pic 15: Tooth after one weak

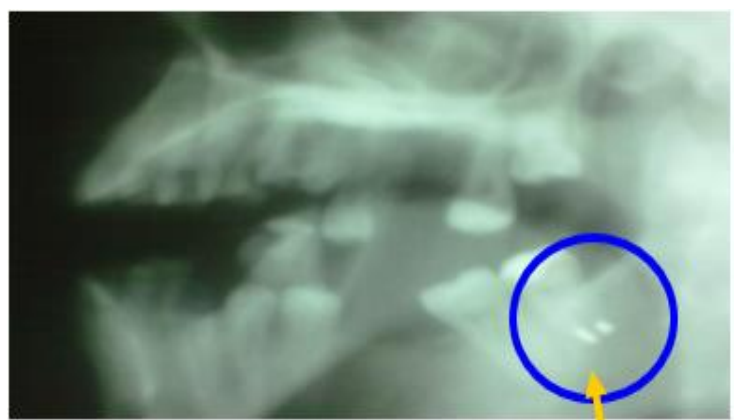

Pic 16: Tooth after 4 months no sign of ankylosis or resorption

\section{Discussion}

Intentional replantation has some advantages over endodontic surgery (apical surgery), which include being easier than apical surgery, less-invasive, less time-consuming, and less-costly procedure. Also, root canal therapy is better-performed extra orally. $(25,26)$ On the other hand, the greatest disadvantage of intentional replantation, which leads most dentists to consider this technique as a last resort to save a tooth, is that replacement resorption or ankylosis may occur. However, recent long-term follow-up studies $(27,28,29)$ have shown that the success rates for intentional replantation are similar to those for apical surgery. The indications for intentional replantation include failure of root canal treatments, anatomic limitations, perforations in areas inaccessible to surgery, persistent chronic pain, or patient management $(30,31)$ In these reported cases which deal with a different patient, Intentional replantation was chosen as the treatment option based on the clinical indication and the patient's refusal to undergo a periapical surgery or retreatment $\mathrm{R}, \mathrm{C}$ filling. The follow-up until now confirmed the successful management of these cases. Splinting is necessary after replantation to reduce the mobility of the tooth and aid the initial periodontal healing. However, replanted teeth should be splinted only for a short period (1 to 2 weeks) $(32,33)$. Splinting was removed 2 weeks after replantation. In these cases, a different type of fixation had been used such as S.S wire, L.C, and glass ionomer, this different fixation showed that the L.C\& Glass ionomer more comfortable to the patients and the gingival healed quickly than that with S.S wire.

The Authors reported principles which essential to the success of IR. They that:

$\&$ The success of this treatment was primarily dependent upon the maintenance of aseptic conditions during the intervention, atraumatic extraction, minimal manipulation of the periodontal ligament, minimizing occlusal forces following replantation, as well as carefully controlled postoperative patient compliance $[34,35]$

In presented cases, this is achieved through chlorhexidine mouth wash and disinfection of the operative field, a careful extraction, gentle removal of the lesion, decrease the height of the occlusal surface of the tooth and give the patient wide 
spectrum antibiotic with analgesia.

\& Furthermore, the removal of all tissue debris and irritating substances from the root surface, as well as achievement of a good apical seal by root-end resection, root-end preparation and root-end filling, are necessary ${ }^{[36]}$. In the present cases, the lesion removed from the apical area, and a tight apical barrier was created, this was done by amalgam which seals the pathways of communication between the infected root canal system and the periradicular tissues.

\& other said that one key factor for success is to limit the damage to the cementum layer of the tooth [37], because regeneration of the PDL is very important to the survival of the tooth, and ankylosis can result if the tooth is retained extraorally for a long period. Studies have shown that teeth that are protected in a physiological ideal media can be replanted within 15 minutes to one hour. Extraoral time (tooth outside of socket) should be kept to a minimum to avoid dehydration and necrosis of ligament ${ }^{[38]}$. In these reported cases, time does not exceed more than half-hour.

\& Careful avoidance of any form of trauma during extraction and reinsertion is also important for treatment success. Trauma to any of the tissues can become an additional cause of impaired healing. An atraumatic surgical technique preserves bone and periodontal support ${ }^{[38]}$. Atraumatic teeth extraction was employed in represented cases.

At last root, resorption and ankylosis may be detectable within 3-4 weeks and 1-12 months, respectively ${ }^{[40]}$. No signs of ankylosis or inflammatory resorption were recorded during follow up of reported cases.

\section{Conclusion}

Intentional replantation can be an alternative treatment option for teeth with hop less or poor prognosis where other options are not possible or refused from the patient and he or she desires to keep the tooth. This technique may help to restore the natural tooth in function and this idea more comfortable to the psychology of the patient as a natural tooth retains to function. Clinical and radiographic follow-ups should be carried out. More extensive studies are necessary.

\section{References}

1. Weinberger B. Introduction to the history of dentistry. St. Louis: CV Mosby, 1948, 105

2. Bender IB, Rossman LE. Intentional replantation of endodontically treated teeth. Oral Surg Oral Med Oral Pathol 1993;76(5):623-30

3. see ref. no. 2

4. Nuzzolese E, Cirulli N, Lepore MM, D'Amore A. Intentional dental reimplantation: a case report. J Contemp Dent Pract 2004;5:121-30.

5. Chandra RV, Bhat KM. Twenty-year follow-up of an unconventional intentional replantation. J Can Dent Assoc 2006;72:639-42.

6. YU. XU B, Wu B Treatment of combined endodonticperiodic lesions by intentional replantation and application of hydroxyapatite, Dent Traumatol 2003;19 (1):60-3

7. Peer M. Intentional replantation-a 'last resort' treatment or a conventional treatment procedure? Nine case reports. Dent Traumatol 2004;20:48-55

8. Grossman LI. Intentional replantation of teeth. J Am Dent Assoc 1966;72:1111-8.

9. Weine FS. The case against intentional replantation. J Am Dent Assoc 1980;100:664-8.

10. Bittencourt GS, Almeida FX. Roldi A. Intentional replantation with tooth rotation as an indication for treatment of crown-root fractures. Revista da SBTD 2009;1:2-6.

11. Kratchman S. Intentional replantation. Dent Clin North Am 1997;41:603-7.

12. Demiralp B, Nohutcu RM, Tepe DI, Eratalay K. Intentional replantation for periodontally involved hopeless teeth. Dent Traumatol 2003;19:45-51.

13. see ref. no. 2

14. see ref. no. 4

15. HTTP:// ENDOSPOT com. Intentional - reimplantation to Manage Persistent Endodontic Disease Without Apical Surgery-Endo Spot 2011

16. previous ref.

17. Deeb E, Prietto P, McKensa R. Replantation of luxated teeth in humans. J South Calif Dent Assoc 1965;33:194206.

18. Grossman L, Ship I. Survival rate of replanted teeth. Oral Surg 1970; 29:899-906.

19. see ref. no.7

20. see ref. no. 15

21. Andreasen JO, Borum MK, Jacobsen HL, Adreasen FM. Replantation of 400 avulsed permanent incisors.4. Factors related to periodontal ligament healing. Endod Dent Traumatol 1995;11:76-83.

22. previous ref.

23. Asgary S. Management of a hopeless mandibular molar: IEJ Iranian Endodontic Journal 2011;6(1):34-37

24. see ref. no. 15

25. see ref. 11

26. see ref.no,7

27. Benenati FW. Intentional replantation of a mandibular second molar with long-term follow-up: report of a case. Dent Traumatol 2003;19:233-6

28. Shintani S, Tsuji M, Toyosawa S, Ooshima T. Intentional replantation of an immature permanent lower incisor because of a refractory periapical lesion: case report and 5-year follow-up. Int J Ped Dent 2004;14:218-22

29. see ref. no.5

30. Dumsha TC, Gutman JC. Clinical guidelines for intentional replantation. Compend Contin Educ Dent $1985 ; 6: 604-8$

31. see ref. no 7

32. see ref. no.7

33. see ref. no. 28

34. Michael R. Cotter, John Panzarino. Intentional replantation: a case report. JOE 2006;32(6):579-582.

35. RAJI- B Intentional Replantation: A Measure to Save the Natural Tooth -Journal of Clinical and Diagnostic Research. 2010;(4):3276-3278

36. Sritharan A. Discuss that the coronal seal is more important than the apical seal for endodontic success. Aust Endod J 2002;28:112-5. Review.

37. see ref, no. 15

38. see ref. no. 21

39. Mendes RA, Rocha G. Mandibular third molar auto transplantation-literature review with clinical cases. J Can Dent Assoc 2004;70:761-6. see ref. no.21. 